\title{
Avaliação da implantação de busca ativa de reações adversas a medicamentos com auxílio de ferramentas informatizadas
}

\author{
Evaluation of the implementation of computerized tools for adverse \\ drug reactions active surveillance
}

Recebido em: 22/11/2016

Aceito em: $\quad 09 / 06 / 2017$
Janaína Martins BRETAS ${ }^{1}$; Maria das Dores Graciano SILVA²; Mariana Martins Gonzaga NASCIMENTO ${ }^{3}$

${ }^{1}$ Oncoclínicas do Brasil. Rua Roma, 26-4 andar. Bairro Santa Lúcia, CEP 30360-680. Belo Horizonte, MG, Brasil. ${ }^{2}$ Hospital das Clínicas. Universidade Federal de Minas Gerais (HC-UFMG). Av. Professor Alfredo Balena, 110, Bairro Santa Efigênia, CEP 30130-100. Belo Horizonte, MG, Brasil. ${ }^{3}$ Programa de Pós-Graduação em Medicamentos e Assistência Farmacêutica.Faculdade de

Farmácia, Universidade Federal de Minas Gerais (PPGMAF/FAFAR/UFMG). Av. Presidente Antônio Carlos, 6627, Campus Pampulha, CEP 31270-901. Belo Horizonte, MG,Brasil.E-mail: marianamgn@yahoo.com.br

\section{ABSTRACT}

The objective of this study was to assess the implementation of adverse drug reactions (ADR) active surveillance strategies using computerized tools in a pharmacovigilance program of a large private hospital. The study was developed in three phases: I. implementation of the pharmacovigilance service (passive surveillance - intensified voluntary notification); II. active surveillance based on managerial reports of consumption of tracer drugs; III. active surveillance based on the content of forms that were filled out in the electronic system whenever a tracer drug was prescribed. All the ADR detected by the pharmacovigilance service for three and a half years $(n=542)$ were analyzed. The difference between the ADR/100 patients-day was analyzed by the comparison of two proportions, with a $5 \%$ significance level and power of $80 \%$. The rate of ADR $/ 100$ patient-day identified in phase II and III ( 0.26 and 0.15 , respectively), that is, after the implementation of computerized tools, was higher than the rate detected in phase I $(0.10)$. The difference between the proportions was statistically significant. The majority of ADR identified presented moderate severity $(69 \%)$, and an increase in this proportion was observed after the implementation of computerized tools. Regarding the causality assessment, $83.6 \%$ of the identified ADR was classified as "possible causality". The implementation of active surveillance using computerized tools provided a statistically significant increase in the identification of ADR identification, reinforcing the need to intensify such pharmacovigilance strategies.

Keywords: Pharmacovigilance; Drug-Related Side Effects and Adverse Reactions; Adverse Drug Reaction Reporting Systems; Electronic Prescribing

\section{RESUMO}

O objetivo do presente trabalho foi avaliar a implantação de estratégias de busca ativa de reações adversas a medicamentos (RAM) com o auxílio de ferramentas informatizadas em um programa de farmacovigilância de um hospital privado de grande porte. O desenvolvimento do estudo se deu em três fases: I - implantação do serviço de farmacovigilância (vigilância passiva - notificação voluntária intensificada); II - busca ativa com base em relatórios gerenciais de consumo de medicamentos rastreadores; III - busca ativa com base no preenchimento de formulários vinculados à prescrição de medicamentos rastreadores. Foram analisadas todas as RAM detectadas pelo serviço por três anos e 
meio $(n=542)$. A diferença entre as taxas de RAM/100 pacientes-dia foi analisada por meio de teste de comparação de duas proporções, com nível de significância de $5 \%$ e poder de $80 \%$. A taxa de RAM/100 paciente-dia encontrada na fase II e III $(0,26$ e 0,15 , respectivamente), ou seja, após a implementação das ferramentas informatizadas, foi superior àquela detectada na fase $\mathrm{I}(0,10)$. A diferença entre as proporções foi estatisticamente significativa. A maioria das RAM identificadas apresentou gravidade moderada (69\%) e observou-se a elevação desta proporção após a implantação das ferramentas informatizadas. Quanto ao perfil de relação causal, 83,6\% das RAM identificadas se enquadravam no critério de "causalidade possível". A implantação da busca ativa utilizando ferramentas informatizadas proporcionou um aumento na identificação de RAM estatisticamente significativo, reforçando a necessidade de intensificar tais estratégias de farmacovigilância.

Palavras-chave: Farmacovigilância; Efeitos Colaterais e Reações Adversas Relacionados a Medicamentos; Sistemas de Notificação de Reações Adversas a Medicamentos; Prescrição Eletrônica.

\section{INTRODUÇÃO}

Todo medicamento pode ocasionar uma resposta nociva e não intencional, mesmo quando utilizado em doses normalmente recomendadas para terapêutica, prevenção ou diagnóstico (1). Estes eventos nocivos são denominados Reações Adversas a Medicamentos (RAM) e sua elevada incidência representa um grande impacto nos sistemas de saúde. Estudos mostram que $2 \%$ a $30 \%$ das admissões em serviços de emergência estão relacionadas à ocorrência de $\operatorname{RAM}(2,3)$. Além disso, estes eventos também são responsáveis por uma parcela significativa dos custos durante a hospitalização e podem aumentar o tempo de internação e a mortalidade (3).

Dentro desse contexto, fazem-se necessários estudos pós-comercialização ou de farmacovigilância que assegurem o monitoramento constante da segurança dos medicamentos aprovados pelas agências reguladoras de cada país (1). No entanto, apesar do importante papel de relatos espontâneos de RAM para sua viabilização, em virtude das elevadas taxas de subnotificação, grande parte dos serviços de farmacovigilância opta por operacionalizar a detecção de eventos adversos utilizando não só notificações voluntárias, mas também a busca ativa (4-6).

Estudos sugerem que a adoção de sistemas de busca ativa em farmacovigilância eleva o potencial de detecção de RAM entre pacientes hospitalizados, e que sua eficiência pode ainda ser otimizada com a aplicação de ferramentas informatizadas para sua operacionalização (4). No entanto, estudos que descrevem a experiência brasileira com a implantação deste perfil de busca de RAM são escassos (7). Tendo isto em vista, o presente trabalho tem como objetivo avaliar a implantação da busca ativa de RAM, com o auxílio de ferramentas informatizadas, em um serviço de farmacovigilância de um hospital privado de grande porte.

\section{MÉTODO}

Trata-se de um estudo observacional descritivo e retrospectivo realizado em um hospital privado de grande porte (300 leitos) e alta complexidade da cidade de Belo Horizonte, Minas Gerais, Brasil. Para tal, foi realizada a análise retrospectiva de fonte secundária composta por um banco de dados contendo todas notificações de RAM do serviço de farmacovigilância da instituição.

O Serviço de Farmacovigilância da Instituição. O serviço de farmacovigilância atende todos os setores do hospital (incluindo unidades de cuidado intensivo), exceto a unidade ambulatorial de oncologia, e contava, à época do estudo, com cinco acadêmicos de farmácia coordenados por um farmacêutico.

No serviço, as RAM detectadas são estratificadas quanto ao critério de causalidade e gravidade de acordo com o algoritmo de Naranjo e cols (1981). Este algoritmo, criado na década de 1980 e adaptado na década de 1991, tem como objetivo avaliar critérios que aumentam ou reduzem a probabilidade de relação causal entre a utilização de um dado medicamento e uma RAM. Ele apresenta 10 questões objetivas que devem ser respondidas pelo avaliador da RAM por meio da seleção de respostas 'sim', 'não" ou 'sem informação'. De acordo com a resposta do avaliador, uma pontuação é conferida a cada uma das questões, gerando um score final que leva à classificação final do perfil da RAM como a) definida (pontuação maior ou igual a 9); b) provável (5 a 8); c) possível (1 a 4); ou d) duvidosa (menor ou igual a 0) (8).

As atividades do serviço de farmacovigilância foram iniciadas na instituição em julho de 2007. Um ano após a implantação do serviço, o uso de ferramentas informatizadas foi iniciado para auxiliar na busca ativa de RAM. A evolução do processo de farmacovigilância na instituição ocorreu em três fases: 
I - Vigilância passiva intensificada

II - Busca ativa com base em relatórios gerenciais de consumo de medicamentos rastreadores

III - Busca ativa com base em documentos de prontuário vinculados à prescrição de medicamentos rastreadores.

Fase I. Vigilância passiva intensificada. Em julho de 2007, foi iniciada a implantação do serviço de farmacovigilância por meio de método de vigilância passiva intensificada. Toda a equipe de enfermagem foi capacitada com intuito de intensificar a detecção de RAM via notificações voluntárias dos profissionais de saúde da instituição. A partir de setembro do mesmo ano, um acadêmico de farmácia foi designado para fazer uma visita semanal a todos os enfermeiros das unidades assistenciais com a finalidade de captar notificações de suspeitas de RAM ocorridas durante o período.

Fase II. Busca ativa com base em relatórios gerenciais de consumo de medicamentos rastreadores. A fase II ocorreu no período de julho de 2008 a junho de 2010. Durante esse período, de segunda a sexta, um acadêmico de farmácia emitia o relatório gerencial de consumo de medicamentos designados como rastreadores (Quadro 1). Estes medicamentos rastreadores foram baseados na padronização da instituição, na experiência obtida na fase inicial do estudo e nos critérios propostos pelo Institute for Healthcare Improvement (IHI) (9).
Os prontuários dos pacientes usuários de medicamentos rastreadores eram analisados com o objetivo de detectar informações que pudessem justificar a prescrição do medicamento rastreador. Caso uma suspeita de RAM fosse identificada, procedia-se com sua notificação e documentação interna.

Fase III. Busca ativa com base em documentos de prontuário vinculados à prescrição de medicamentos rastreadores. A fase III ocorreu de junho de 2010 a dezembro de 2010 após a inserção de um formulário ao sistema informatizado de prescrição. Esse documento, de preenchimento obrigatório pelo médico, foi vinculado à primeira prescrição de cada um dos medicamentos designados como rastreadores (Quadro 1).

Um acadêmico de farmácia ficou como responsável pela impressão e análise, de segunda à sexta, do relatório com todos os documentos de busca ativa de RAM com sinalização positiva para suspeita de RAM. Os prontuários destes pacientes eram então analisados retrospectivamente para identificar se havia uma possível RAM vinculada ao uso de algum medicamento prescrito durante a internação hospitalar.

Mensuração e caracterização de RAM. Foram incluídas na análise do presente estudo todas as RAM detectadas pelo serviço de farmacovigilância do hospital referente aos meses de julho de 2007 a dezembro de 2010, totalizando 542 RAM. As suspeitas de RAM iden-

Quadro 1. Medicamentos utilizados como rastreadores de Reações Adversas Relacionadas a Medicamentos pelo serviço de farmacovigilância da instituição em estudo - Belo Horizonte. 2008 a 2010.

\begin{tabular}{|ll}
\hline Principio Ativo do Medicamento Rastreador & Apresentação \\
\hline Cloreto de potássio & Comprimido simples - $600 \mathrm{mg}$ \\
\hline Dexclorfeniramina & Comprimido simples $-2 \mathrm{mg}$ \\
\hline Fexofenadina & Comprimido simples $-180 \mathrm{mg}$ \\
\hline Fitomenadiona & Solução injetável (IM) $-10 \mathrm{mg} / \mathrm{mL}$ Ampola de $1 \mathrm{~mL}$ \\
& Solução injetável (IV) $-10 \mathrm{mg} / \mathrm{mL}$ Ampola de $1 \mathrm{~mL}$ \\
\hline Flumazenil & Solução injetável $-2 \mathrm{mg} / 0,2 \mathrm{~mL}$ Ampola de $0,2 \mathrm{~mL}$ \\
\hline Glucagon & Solução injetável $-0,5 \mathrm{mg} / 5 \mathrm{~mL}$ \\
\hline Gliconato de cálcio & Ampola de $5 \mathrm{~mL}$ \\
\hline Hidroxizina & Solução injetável $-1 \mathrm{mg} / \mathrm{mL} \mathrm{Ampola} \mathrm{de} 1 \mathrm{~mL}$ \\
\hline Naloxona & Solução injetável $-10 \% \mathrm{Ampola} \mathrm{de} 10 \mathrm{~mL}$ \\
\hline Ondansetrona & Cápsula - $25 \mathrm{mg}$ \\
\hline Poliestirenossulfonato de cálcio & Solução injetável $-0,4 \mathrm{mg} / \mathrm{mL}$ Ampola de $1 \mathrm{~mL}$ \\
\hline Sulfato de Protamina & Solução injetável $-8 \mathrm{mg} / 4 \mathrm{~mL}$ Ampola de $4 \mathrm{~mL}$ \\
\hline
\end{tabular}


tificadas pelo serviço de farmacovigilância do hospital foram caracterizadas e agrupadas de acordo com seu perfil de causalidade e gravidade. Não foram incluídos, na análise, erros de medicação ou desvios de qualidade.

As informações foram extraídas retrospectivamente do banco de dados do sistema da qualidade do hospital em estudo e apresentados sob a forma de taxa de RAM por 100 pacientes-dia. Os dados foram seccionados de acordo com as fases do estudo.

Análise Estatística. A diferença entre as taxas de incidência de RAM/100 pacientes dia foi analisada por meio de teste de comparação de duas proporções, com nível de significância de $5 \%$ e poder de $80 \%$, utilizando-se o software Primer of Biostatistics 5.

Aspectos éticos. Esse estudo foi aprovado pelo Comitê de Ética em Pesquisa do Hospital Mater Dei, em 24 de novembro de 2010. Durante sua execução, foram respeitados os preceitos éticos em pesquisa com seres humanos, de acordo com a Resolução no 196/1996 do Conselho Nacional de Saúde.

\section{RESULTADOS E DISCUSSÃO}

Mensuração das RAM. Durante a fase I foi detectada uma taxa média de $0,10 \mathrm{RAM} / 100$ pacientes-dia ( $\mathrm{n}=77$ RAM). Após a implantação do processo de busca ativa com base em relatórios gerenciais de consumo de medicamentos rastreadores (fase II), este indicador apresentou um valor médio de $0,26 / 100$ pacientes-dia ( $\mathrm{n}=$ 384 RAM). Após a implantação da fase III, foram identificadas 71 RAM, correspondendo a uma a taxa média de $0,15 \mathrm{RAM} / 100$ paciente-dia. Neste período final, os prescritores da instituição sinalizaram 3,5 RAM para cada 100 medicamentos rastreadores prescritos. Durante todo o período de análise do presente estudo (fase I, II e
III), foram realizadas apenas dez relatos espontâneos de RAM provenientes de médicos

A análise estatística demonstrou que todas as proporções de RAM/100 pacientes-dia eram diferentes quando comparadas entre si $(\mathrm{p}<0,05)$.

Caracterização das RAM. A maioria das RAM identificadas $(68,9 \%)$ durante o período total de estudo apresentou gravidade moderada. No entanto, foi observada uma tendência à elevação da proporção de RAM de gravidade moderada em relação às demais, após a implantação das ferramentas informatizadas de busca ativa (Tabela 1). Quanto ao perfil de relação causal, 83,6\% das RAM identificadas durante o estudo se enquadraram no critério de "causalidade possível" (Tabela 1).

Na primeira etapa do estudo (Fase I), o método de vigilância passiva intensificada esteve associado a um número reduzido de notificações $(\mathrm{n}=77$; média de 40 RAM notificadas/ano), porém superior ao detectado em um estudo brasileiro realizado em um hospital de 133 leitos onde foi adotado o método de vigilância passiva não intensificada (média de 19,6 RAM notificadas/ano) (10). Por outro lado, é importante destacar que, apesar do volume reduzido de notificações, uma elevada proporção de RAM classificadas como "definidas" foram detectadas durante a Fase I, mostrando que profissionais de saúde tendem a notificar ativamente reações que pressupõem uma conexão mais direta entre o uso do medicamento e o evento. Isso indicou a necessidade de informar aos profissionais que é possível e encorajado notificar RAM potenciais mesmo quando ainda não há certeza quanto ao seu perfil de causalidade, e apresentar o papel da equipe do serviço de farmacovigilância na avaliação desses critérios de forma mais aprofundada.

Nas etapas subsequentes do presente estudo, foi observado um impacto positivo da implantação de ferra-

Tabela 1. Frequência relativa de Reações Adversas Relacionadas a Medicamentos (RAM) identificadas nas diferentes fases de implantação do serviço de farmacovigilância da instituição em estudo conforme, critério de causalidade e gravidade (Belo Horizonte. 2007 a 2010)

\begin{tabular}{|c|c|c|c|c|c|c|}
\hline \multirow{2}{*}{$\begin{array}{l}\text { Implantação do serviço de } \\
\text { farmacovigilância (método notificação/ } \\
\text { detecção de } \text { RAM }^{*} \text { ) }\end{array}$} & \multicolumn{3}{|c|}{$\begin{array}{l}\text { Frequência relativa das } \text { RAM }^{*} \text { identificadas } \\
\text { conforme a gravidade** }(\%)\end{array}$} & \multicolumn{3}{|c|}{$\begin{array}{l}\text { Frequência relativa das RAM* identificadas } \\
\text { conforme a causalidade** }(\%)\end{array}$} \\
\hline & Leve & Moderada & Grave & Possivel & Provável & Definida \\
\hline $\begin{array}{l}\text { Fase II (busca ativa - relatório de consumo de } \\
\text { medicamentos marcadores) }\end{array}$ & 7,8 & 68,5 & 23,7 & 90,6 & 9,1 & 0,3 \\
\hline $\begin{array}{l}\text { Fase III (busca ativa - documentos de } \\
\text { prontuário vinculado à prescrição de } \\
\text { medicamentos marcadores) }\end{array}$ & 1,4 & 88,7 & 9,9 & 90,1 & 9,9 & 0,0 \\
\hline Fase I, II e III & 13,4 & 68,9 & 17,7 & 83,6 & 15,4 & 1,0 \\
\hline
\end{tabular}


mentas informatizadas na intensificação da detecção de RAM pelo setor de farmacovigilância. Mediante a análise das taxas de RAM/100 pacientes-dia, foi observado um aumento na detecção de RAM após a implantação de rastreadores associados às ferramentas informatizadas, sendo que a implantação da fase II teve maior impacto na elevação da taxa que a fase III. O aumento na proporção de RAM detectadas mostrou um melhor desempenho da busca ativa quando comparado à notificação voluntária, reforçando a necessidade de adoção desta metodologia para aumentar a efetividade dos serviços de farmacovigilância.

Em uma revisão sistemática, foi avaliada a eficiência de diferentes métodos de busca por problemas relacionados a medicamentos, e, assim como no presente trabalho, os resultados obtidos também mostraram que o uso de ferramentas rastreadoras foi mais efetivo que o método de relatos voluntários em identificar eventos, mesmo quando este último estava associado a estratégias intensificadoras (11). Embora métodos observacionais diretos e revisões de prontuários tenham sido mais eficazes do que as ferramentas rastreadoras, estes proporcionaram custo mais elevados (11). Uma outra revisão sistemática mostrou que o uso de ferramentas informatizadas dobrou o número de notificações de RAM em hospitais, e apontou sua eficiência e importância para facilitar a operacionalização da farmacovigilância (12). No entanto, ambas revisões apontaram que, apesar do número de estudos descrevendo o uso de tecnologias para aprimorar a notificação de RAM tenha aumentado ao longo dos anos, poucos deles descreveram quantitativamente as notificações de RAM antes e após implementação de tecnologias em saúde, como foi descrito no presente estudo $(11,12)$.

Embora fosse esperado um aumento da taxa de notificações após a implantação da ferramenta de busca ativa da Fase III, a expectativa não se confirmou, sendo que o número de RAM detectadas com este método correspondeu a pouco mais que $10 \%$ dos valores apurados por Rozich e cols. (2003), que descreveram o uso concomitante de ferramentas rastreadoras e do método de busca de eventos adversos por revisão de prontuário em 86 hospitais (28 RAM para cada 100 medicamentos rastreadores prescritos) (13). A discrepância entre estes números indicou uma potencial subnotificação e revelou a necessidade de manter ativa a estratégia de análise de prontuários dos pacientes em uso de medicamentos rastreadores, adotando desta forma, as estratégias de busca ativa das Fases II e III propostas no presente estudo.
Esta subnotificação identificada pode ser decorrente do baixo grau de informação do corpo clínico quanto ao serviço de farmacovigilância estabelecido na instituição. Além disso, muitos profissionais queixam-se do excesso de formulários, alertas e justificativas que precisam ser visualizados e preenchidos no ato da prescrição. Estas questões também são apontadas por estudos que avaliaram a atitude de profissionais de saúde quanto à notificação de RAM, sendo destacado que o processo de notificação deve ser simples $(14,15)$ e o conhecimento acerca da sua importância e do papel dos serviços de farmacovigilância deve ser intensificado por meio de estratégias de qualificação da equipe institucional $(15,16)$. Este cenário reforça a necessidade da participação do farmacêutico nas reuniões clínicas das equipes de saúde com o objetivo de apresentar o serviço de farmacovigilância e a importância das ferramentas informatizadas para a segurança do paciente. Além disso, devem ser adotadas medidas adicionais como a divulgação dos critérios de notificação de RAM junto aos profissionais de saúde da instituição, bem como do perfil e frequência das RAM detectadas na instituição com o intuito de prevenir sua recorrência. No entanto, o fato do hospital possuir corpo clínico aberto pode vir a limitar a efetividade destas ações.

A tendência à elevação da proporção de RAM de gravidade moderada em relação às demais após a implantação das ferramentas informatizadas de busca ativa era esperada, uma vez que os rastreadores utilizados neste processo, em sua maioria, são antídotos ou medicamentos frequentemente utilizados no tratamento de RAM inespecíficas como náuseas e alterações dermatológicas. Portanto, as reações apontadas por esta ferramenta, tendem a apresentar gravidade minimamente moderada, uma vez que o conceito de RAM leve, segundo Naranjo e cols (1981), compreende eventos que não necessitaram de tratamentos específicos (8). Por outro lado, o fato de grande parte dos pacientes hospitalizados estarem sob tratamento medicamentoso ou não medicamentoso de um ou mais problemas de saúde, faz com que, na maioria das vezes, existam alternativas plausíveis para a ocorrência de eventos adversos, justificando a predominância de reações classificadas como "possivelmente relacionadas a medicamento" entre as RAM identificadas no presente estudo.

A carência de estudos com desenhos semelhantes na literatura brasileira limita a análise comparativa dos resultados entre hospitais de perfis semelhantes. Além disso, o presente estudo tem como limitação ser referen- 
te a um período histórico retrospectivo (2007 a 2010), apesar de extenso. No entanto, acredita-se que este fator não reduza a importância da divulgação de tais dados, uma vez que o perfil da assistência farmacêutica hospitalar é variado no Brasil, e os resultados encontrados podem proporcionar subsídio para que novos serviços institucionais de farmacovigilância sejam implantados e avaliados nas organizações hospitalares brasileiras, sobretudo em instituições que ainda se encontram no processo de implantação de ferramentas informatizadas.

\section{REFERÊNCIAS}

1. OMS. Organização Mundial da Saúde. Estrutura conceitual da classificação internacional sobre segurança do doente: relatório técnico final. Geneva: WHO; 2011. Disponível em: http://apps.who.int/iris/bitstream/10665/ 70882/4/WHO_IER_PSP_2010.2_por.pdf?ua=1

2. Hakkarainen KM, Hedna K, Petzold M, Hagg S. Percentage of patients with preventable adverse drug reactions and preventability of adverse drug reactions--ameta-analysis. PLoS One. 2012; 7(3):e33236. DOI: 10.1371/ journal.pone.0033236.

3. Sultana J, Cutroneo P, Trifirò G. Clinical and economic burden of adverse drug reactions. J Pharmacol Pharmacother. 2013; 4(Suppl1):S73-S77. DOI: 10.4103/0976500X.120957.

4. Coloma PM, Trifirò G, Patadia V, Sturjenboom M. Postmarketing safety surveillance: where does signal detection using electronic healthcare records fit into the big picture? Drug Saf. 2013; 36(3):183-197. DOI: 10.1007/ s40264-013-0018-x.

5. Bates DW, Evans RS, Murff H, Stetson PD, Pizziferri L, Hripcsak G. Detecting Adverse Events Using Information Technology. J Am Med Inform Assoc. 2003; 10(2):115-128.

6. Dormann H, Muth-Selbach U, Krebs S, Criegee-Rieck M, Tegeder I, Schneider HT, Hahn EG, Levy M, Brune $\mathrm{K}$, Geisslinger G. Incidence and Costs of Adverse Drug Reactions During Hospitalisation Computerised Monitoring Versus Stimulated Spontaneous Reporting. Drug Saf. 2000; 22(2):161-168.

7. Cano FG, Rozenfeld S. Adverse drug events in hospitals: a systematic review. Cad Saude Publica. 2009; 25(2):s360-s372.

8. Naranjo CA, Busto U. Reações adversas às drogas. In: Kalant H, Roschlau WHE. Princípios de farmacologia médica. Rio de Janeiro: Guanabara Koogan, 1991. P. 537-542.

9. Griffin FA, Resar RK. IHI Global Trigger Tool for Measuring Adverse Events. In: IHI Innovation Series white paper. Disponível em: http://www.ihi.org/resources/pages/ihiwhitepapers/ihiglobaltriggertoolwhitepaper.aspx

\section{CONCLUSÃO}

A implantação da busca ativa utilizando ferramentas informatizas proporcionou expressivo aumento na identificação de RAM na instituição, reforçando a necessidade de adotar ou intensificar tal abordagem quando possível. Na instituição em estudo, o uso de ferramentas informatizadas para identificação de RAM continua a ser aplicada e envolve diretamente a atuação dos profissionais farmacêuticos no seu contínuo aprimoramento.

10. Pereira ST, Batista PN, Gomes KKLB, Freitas RM, Nunes LCC. Avaliação das notificações de eventos adversos a medicamentos de um hospital de Picos - Piauí. Rev Bras Farm Hosp Serv Saúde. 2012; 3(3):18-22.

11. Meyer-Massetti CM, Cheng CM, Schwappach DL, Paulsen L, Ide B, Meier CR, Guglielmo BJ. Systematic review of medication safety assessment method. Am J Health Syst Pharm. 2011; 68(3): 227-240. DOI: 10.2146/ ajhp100019.

12. Ribeiro-Vaz I, Silva AM, Costa Santos C, Cruz-Correia R. How to promote adverse drug reaction reports using information systems - a systematic review and meta-analysis. BMC Med Inform Decis Mak. 2016. 16:27. DOI: 10.1186/s12911-016-0265-8.

13. Rozich JD, Haraden CR, Resar RK. Adverse drug event trigger tool: a pratical methodology for measuring medication related harm. Qual Saf Health Care. 2003; 12(3):194-200.

14. Peymani P, Tabrizi R, Afifi S, Namazi S, Heydari ST, Shirazi MK, Nouraei H, Sadeghi E, Lankarani KB, Maharlouei N. Knowledge, attitude and practice of General Practitioners towards adverse drug reaction reporting in South of Iran, Shiraz. Int J Risk Saf Med. 2016;28(1):2531. DOI: $10.3233 / J R S-160670$.

15. Mendes Marques JI, Polónia JM, Figueiras AG, Costa Santos CM, Herdeiro MT. Nurses' attitudes and spontaneous adverse drug reaction reporting: a case-control study in Portugal. J Nurs Manag. 2016;24(3):409-416. DOI: 10.1111/jonm. 12337.

16. De Angelis A, Colaceci S, Giusti A, Vellone E, Alvaro R. Factors that condition the spontaneous reporting of adverse drug reactions among nurses: an integrative review. J Nurs Manag. 2016; 24(2):151-163. DOI: 10.1111/ jonm. 12310. 